\section{Análise espacial da violência doméstica contra a mulher entre os anos de 2002 e 2005 em João Pessoa, Paraíba, Brasil}

\author{
Spatial analysis of domestic violence against \\ women from 2002 to 2005 in \\ João Pessoa, Paraíba State, Brazil
}

Kerle Dayana Tavares de Lucena 1 Ana Tereza Medeiros Cavalcanti da Silva 1 Ronei Marcos de Moraes 1 César Cavalcanti da Silva 1 Italla Maria Pinheiro Bezerra 1

\footnotetext{
1 Universidade Federal da Paraíba, João Pessoa, Brasil.

Correspondence

K. D. T. Lucena

Universidade Federal da

Paraíba.

Rua Pedro Batista da Silva 102, João Pessoa, PB 58040-510, Brasil.

kerledayana@yahoo.com.br
}

\section{Abstract}

This was a quantitative, ecological, exploratory study using spatial analysis and a geographic information system. The population consisted of all the women that had filed complaints against domestic violence in the city of João Pessoa, Paraíba State, Brazil, from 2002 to 2005, at the Specialized Police Precinct for Women. The aim was to investigate the spatial distribution of domestic violence in order to assist policymakers in the decisionmaking process. Analysis of the results allowed identifying areas of low and high incidence of domestic violence, besides comparing the risk in each neighborhood to the overall rate for the city of João Pessoa. Based on the Getis and Ord index, the study produced a decision on priority areas for intervening in domestic violence. The authors highlight the need for changes in care for female victims of violence and the link among institutions with permanent education in the services, including discussions on gender and violence against women.

Domestic Violence; Women; Gender Identity

\section{Introdução}

A área da saúde é constituída por uma complexidade que decorre de sua interseção com as diferentes áreas do conhecimento como a Administração, a Economia, o Direito, a Sociologia, a Estatística, a Geografia, a Informática. Nesse contexto interdisciplinar, a violência de uma forma geral e a violência contra a mulher têm sido reconhecidas como questões que mantêm uma situação de comunicação com todas as áreas do conhecimento humano, por seu caráter transversal a todas as dimensões da experiência de viver de homens e mulheres 1 .

A visibilidade desse fenômeno, a partir do Século XX, foi sintetizada na categoria sociológica conhecida como gênero. Essa categoria ampara tipos de violência decorrentes das relações desiguais entre os sexos. Desse modo, apesar de a violência não ser um tema exclusivo da área de saúde, ele a afeta sobremaneira, pelos danos físicos e psicológicos que ocasiona. A compreensão do impacto da violência na vida individual e social implica envolver, em sua análise, outros âmbitos, como o que se refere às condições socioeconômicas de uma população e às questões ligadas ao estilo de vida, temas relacionados com o território.

Assim, a violência é um fenômeno histórico e social, que apresenta diferentes conteúdos e formas nas diversas sociedades, de acordo com 
a variação de valores culturais que influenciam os juízos éticos de cada uma delas. As diferenças culturais dificultam a conceituação da violência, todavia certos aspectos que a envolve são mantidos, nas diversas sociedades e culturas, permitindo sua caracterização.

Entre as características que mais se recorrem para conceituar a violência de gênero, encontram-se: o emprego da força física (dimensão biológica) e a submissão e a opressão (dimensões psicológica e sociológica). Nessa perspectiva, a violência praticada contra a mulher assume um enfoque diferenciado, uma vez que é praticada por um agressor que compartilha relações íntimas e afetuosas com a vítima. A aplicação da força física e/ou constrangimento psicológico que se impõe a alguma mulher contra seus interesses, vontades e desejos, resulta em danos à saúde física e mental pela violação da dignidade humana em sua integridade. É produzida sob a organização hierárquica do domínio masculino nas relações sociais entre os sexos, historicamente delimitadas, culturalmente legitimadas e cultivadas, nas quais a mulher está exposta a agressões objetivas e subjetivas, tanto no espaço público como no privado 2 .

As consequências da violência, sofrida pela mulher, materializam-se em agravos biológicos, psicológicos, morais e sociais, que dificultam sua experiência de viver a igualdade humana e social plenamente. A persistência e a multiplicidade das formas de expressão dessa espécie de violência, ao longo da história, indicam a importância do tema e a necessidade de se investigar como essa prática interfere no processo de viver, adoecer e morrer de quem as sofre.

Dados mundiais mostram que quase metade das mulheres assassinadas é morta pelo marido ou namorado, atual ou ex. A violência responde por aproximadamente $7 \%$ de todas as mortes de mulheres entre 15 e 44 anos no mundo todo. Em alguns países, até $69 \%$ das mulheres relatam terem sido agredidas fisicamente, e até $47 \%$ declaram que sua primeira relação sexual foi forçada 3 . No Brasil, a violência contra a mulher é um dos maiores problemas de saúde pública, atingindo um quarto da população, excetuando-se os casos não notificados e a violência psicológica que de tão incidente deixou de ser percebida como agressão por não causar danos físicos severos 4 .

De acordo com o Ministério da Saúde 5, uma pesquisa realizada também pela Organização Mundial da Saúde (OMS) no ano de 2002, em oito países, inclusive o Brasil em dois estados (São Paulo e Pernambuco), reconhecem que a morbidade provocada pela violência doméstica e sexual atinge prioritariamente a população feminina, na faixa etária de 15-49 anos. Em Pernambuco,
$34 \%$ das mulheres entrevistadas relataram ter sofrido violência física e/ou sexual por parte do parceiro e apresentaram problemas de saúde decorrentes do episódio, como: dores, desconforto severo, problemas de concentração e tontura; tentativas de suicídio e uso frequente de álcool, configurando a violência como uma questão de saúde física e mental.

A violência contra a mulher na abordagem de gênero considera a diversidade dos processos de socialização de homens e mulheres. Contrapõese ao entendimento do enfoque hegemônico clássico, que "naturalizou" as desigualdades entre os sexos, determinando consequências que impactam a vida e as relações dos seres humanos, tanto no plano individual quanto no coletivo, distanciando a mulher de sua emancipação social. A contradição de gênero é uma das três grandes contradições produzidas na sociedade ocidental. As outras duas são referentes à classe e à raça/etnia 6 .

Quanto ao Estado da Paraíba, dados sobre as causas de óbitos de mulheres em idade fértil (10-49 anos) revelaram os seguintes números, incluindo suicídios, homicídios e lesões intencionais indeterminadas, no ano de 1999: 34 casos no universo de 944 óbitos; no ano 2000: 47 casos no universo de 984 óbitos; no ano de 2001: 52 casos no universo de 1.049 óbitos; e no ano de 2002: 56 casos no universo de 1.0817 .

Os números explicitam a magnitude do problema e as inúmeras consequências à saúde física e mental, além de perdas potenciais no campo do desenvolvimento pessoal, social, afetivo e econômico. A violência perpetrada contra as mulheres é uma das manifestações extremas das relações sociais desiguais entre os sexos. Desse modo, a violência baseada no gênero tem se constituído um fenômeno social que influencia sobremaneira o modo de viver, adoecer e morrer das mulheres 8 .

Considerando a explicação hegemônica sobre a naturalização essencial do feminino e do masculino, ou sobre os gêneros naturalizados, a desigualdade sexual iniciada nos primórdios da história mantém-se, na atualidade, por meio de interesses sociais igualmente naturalizados, constituindo uma espécie de ideologia que subverte a compreensão da realidade essencial do ser humano e obstaculiza a igualdade no âmbito da diversidade. No sentido de superar essa realidade, ao pensamento naturalista opõe-se o que afirmam todos os fenômenos sociais como produtos da ação humana, sendo por isto passíveis de ser por ela também transformados 9 .

A mulher apresenta problemas e necessidades singulares de saúde, diferentes das necessidades dos demais grupos que compõem a esfera 
social. Segundo a Política Nacional de Atenção Integral à Saúde da Mulher, a vulnerabilidade feminina frente a certos agravos está mais relacionada com as questões de gênero - como a sua situação de discriminação na sociedade - do que com fatores biológicos 5 .

Nesse contexto, é pertinente a utilização dos Sistemas de Informações Geográficas (SIG), que são ferramentas computacionais do campo do geoprocessamento, para a montagem de um painel socioterritorial ampliado, que potencializará o olhar dos pesquisadores/gestores sobre os possíveis focos e áreas/fatores de risco de violência doméstica contra a mulher 10 .

A utilização do SIG na análise espacial de dados da saúde pública tem-se destacado nesta área por considerar não apenas a informação, mas, também, o local de ocorrência dos eventos, principalmente na vigilância epidemiológica, avaliação dos serviços de saúde, urbanização e ambiente. O conhecimento produzido é importante para o processo de planejamento da oferta de serviços de saúde e, também, para a avaliação do impacto das ações de saúde 11 .

Os dados de notificação da violência doméstica contra a mulher, no Estado da Paraíba e no Município de João Pessoa, não estão organizados em um banco de dados. Sua organização facilitaria o trabalho dos pesquisadores, no sentido de dar visibilidade ao problema, além de orientar as autoridades a tomarem decisões com o objetivo de prevenir e/ou minimizar os efeitos desse tipo de violência. Os dados notificados na Secretaria de Segurança Pública do Estado da Paraíba, não coincidem com os dados existentes na Secretaria de Saúde do município sobre o tema em questão. Esse desacordo pode estar relacionado à falta de comunicação entre as instituições que compõem a rede de serviços de atenção à mulher em situação de violência 12 . O fato reforça o interesse para se investigar a problemática e agrega importância a este estudo.

Algumas decisões políticas e institucionais foram tomadas para enfrentar a violência contra a mulher no Estado da Paraíba, como a criação de Delegacias da Mulher (seis já instituídas em todo o estado). A cidade de João Pessoa dispõe de uma delas, a Delegacia Especializada de Atendimento da Mulher.

Em termos epidemiológicos há uma série de ocorrências, entre as quais a violência doméstica contra a mulher, que pode ser melhor compreendida e enfrentada quando se articula a saúde com outras áreas do conhecimento, como o meio ambiente. Desse modo, defende-se a intensificação do uso das ferramentas do Geoprocessamento em Saúde Pública para subsidiar o processo de tomada de decisão dos gestores/pesquisadores, na construção de políticas públicas para a atenção à saúde da mulher. Diante da problemática apresentada, questiona-se: como se apresenta espacialmente a violência doméstica contra a mulher em João Pessoa e quais as áreas prioritárias de intervenção?

Para orientar o estudo sobre as questões, formulou-se o seguinte objetivo: investigar a distribuição espacial de violência doméstica contra a mulher para subsidiar os gestores no processo de tomada de decisão nas políticas de saúde da mulher.

\section{Métodos}

Trata-se de um estudo quantitativo, exploratório, ecológico, com a utilização de técnicas de análise espacial de dados de área, modeladas por geo-objeto, uma vez que os dados de violência doméstica contra a mulher estão agregados por bairros no Município de João Pessoa. A população foi composta por todas as ocorrências denunciadas por mulheres vítimas de violência doméstica, residentes em João Pessoa, entre 2002 e 2005, na Delegacia Especializada de Atendimento da Mulher. Ao todo, no período citado, foram registradas 2.836 ocorrências. Nesse sentido, utilizaram-se nesta pesquisa dados de área, considerando que estes dados são usados quando não se dispõe da localização exata do evento ${ }^{13}$. Os dados foram do tipo secundário, coletados por meio do registro das ocorrências de violência contra a mulher, registrados na Delegacia Especializada de Atendimento da Mulher do município cenário do estudo, identificando a incidência da violência doméstica contra as mulheres.

Após a coleta dos dados foi construído um banco de dados para análise estatística, no qual todas as informações relativas a cada caso foram descritas na forma de variáveis. Inicialmente, realizou-se uma análise exploratória para reconhecimento das variáveis e correção de possíveis erros ou inconsistências no preenchimento ou na digitação dos dados. Depois das correções necessárias procedeu-se a análise dos dados, aplicando a estatística descritiva, com a inclusão dos resultados em gráficos.

Nessa perspectiva, os dados de violência foram analisados, considerando os três anos conjuntamente para dar maior respaldo aos resultados. Para processamento e análise, os dados foram inicialmente armazenados numa planilha eletrônica e, logo após, transferidos para o software R (The R Foundation for Statistical Computing, Viena, Áustria; http://www.r-project. org) para análise estatística e análise estatística 
espacial. Os arquivos referentes ao mapa digital da cidade de João Pessoa foram obtidos no Laboratório de Estatística Aplicada ao Processamento de Imagens e Geoprocessamento (LEAPIG) do Departamento de Estatística, Universidade Federal da Paraíba.

A comparação entre os casos nos diversos bairros foi possível usando-se a divisão entre a incidência de violência doméstica contra a mulher específica por bairro pela incidência de violência doméstica contra a mulher para toda a cidade de João Pessoa, confeccionando o mapa de risco relativo, indicador que descreve a intensidade de ocorrência do evento considerado, ou seja, a taxa de incidência (ou risco). Essa é definida como o quociente entre o número de casos ocorridos numa determinada área pela população em risco quanto ao evento 13 .

Para responder ao objetivo deste estudo, foram realizadas as análises de mapas de incidência, risco relativo e índice de aglomeração espacial. Para tanto, verificou-se a normalidade da distribuição dos dados da violência doméstica contra a mulher aplicando o teste de Lilliefors, que pondera os valores centrais e extremos da distribuição. Refere-se ao grau de concordância entre a distribuição de um conjunto de valores amostrais. Com o nível de significância (valor de p) calculado, pode-se aceitar ou rejeitar a hipótese de nulidade $H_{0}$ de que os dados seguem uma distribuição normal. Os dados obtiveram distribuição não normal (valor de $\mathrm{p}<0,05$ ), assim, o teste de escolha para verificar a hipótese de ocorrência de correlação espacial da violência doméstica contra a mulher em João Pessoa não poderia prescindir da normalidade dos dados.

O índice de Getis e Ord (G) é uma medida de associação espacial não paramétrica, cuja função é medir as dependências espaciais. Esse índice pode ser analisado ou aplicado aos dados em que a distribuição não foi normal, com o objetivo de identificar aglomerações espaciais, ou seja, realizações de uma mesma variável aleatória em lugares distintos do espaço 14.

As estatísticas de Getis e Ord são estimadas a partir de grupos vizinhos da distância crítica $d$ de cada área $i$. A distância crítica é formada com base em uma matriz de proximidade $W$, em que cada elemento é dito em função da distância crítica wij (d). Getis e Ord propuseram duas funções estatísticas: a estatística global $G(d)$ que é similar às medidas tradicionais de aglomeração espacial e que se resume a um único valor, e as estatísticas locais $\mathrm{G} i$ e $\mathrm{G} i$ * que são medidas de associação espacial para cada área $i$ 14. A estatística global G(d), para uma determinada distância crítica é dada por:

$$
G(d)=\frac{\Sigma i \Sigma j W i j(d) x i ~ y j}{\Sigma i \Sigma j x i ~ y j}
$$

Em que $x i$ é o valor observado na posição $i$ e wij(d) é um elemento da matriz de proximidade. $\mathrm{O}$ índice global de Getis e Ord fornece um valor que caracteriza a associação espacial existente em toda a região de estudo. Esse índice mede a autocorrelação espacial de todas as áreas que estão a uma distância $d i j$ inferior a $d$. A partir de um nível de significância, que é definido como a probabilidade de rejeitar a hipótese nula - existência de autocorrelação espacial que mede o quanto o valor observado de um atributo numa região é independente dos valores desta mesma variável nas localizações vizinhas - sendo ela verdadeira, o valor-p é confrontado com a significância adotada definindo a significância do índice Getis $e$ Ord gerado.

Sua avaliação é feita com base no valor do índice e de sua significância: o valor positivo e significativo de $G(d)$ indica aglomeração espacial de valores elevados, enquanto valores negativos e significantes de $G(d)$ indicam aglomeração espacial de valores pequenos. Neste estudo, a hipótese que se pretendeu verificar para a construção do modelo de decisão foi: verificar se há aglomerações espaciais da violência doméstica contra a mulher nos bairros do município estudado, para assim, destacarmos as possíveis intervenções. Dessa forma, o índice de Getis e Ord global não nos informa a área exata de aglomeração espacial, por isto, necessitamos calcular o índice local de Getis e Ord, identificado usando-se a seguinte equação:

$G_{i}^{*}(d)=\frac{\sum_{j=1}^{n} w_{i j}(d) x_{j}}{\sum_{j=1}^{n} x_{j}}$

O índice local, que é associado a cada área $A i$, permite uma análise mais detalhada da associação espacial dos dados. O índice de Getis e Ord apresenta um p-valor que testa a significância da estatística gerada. Será considerada significante se o valor de $\mathrm{p}$ for menor que a significância adotada. Neste estudo, adotamos em todos os testes um nível de significância igual a 5\%.

Várias distâncias são testadas para verificar a significância dos resultados no teste global de correlação espacial de Getis e Ord para cada ano. Para os valores significantes, é possível então calcular o teste local de correlação espacial de Getise Ord para o referido ano e distância. A interpretação do índice local é realizada da seguinte forma: valores padronizados positivos e significantes (valor de $\mathrm{p}<5 \%$ ) indicam uma aglomeração espacial de valores altos. Os valores das estatísticas padronizadas negativas e significantes (valor de 
$\mathrm{p}<5 \%$ ) apontam para uma aglomeração espacial de valores baixos.

Para a execução desta pesquisa, consideraram-se as orientações preconizadas pela Resolução $n^{\circ}$. 196/1996, do Conselho Nacional de Saúde, envolvendo seres humanos, atendendo desta forma ao princípio ético da autonomia. Este estudo foi aprovado pelo Comitê de Ética em Pesquisa do Centro de Ciências da Saúde da Universidade Federal da Paraíba, em 28 de novembro de 2007, sob o protocolo de $n^{\circ}$ 024/08.

\section{Resultados}

Buscando visualizar a distribuição espacial dos dados, foram gerados mapas coropléticos. Verificou-se que, conforme o mapa de incidência (Figura 1), os bairros com maior proporção (<1/1.000 habitantes do bairro) nos anos de 2002 a 2005 foram: Ilha do Bispo, Centro, Mandacaru, Bairro São José, Penha e Grotão. Destacam-se também, os bairros do Valentina, Costa e Silva, Bancários e Bessa que apresentaram entre 0,8 e 1 caso para cada mil habitantes. Já os bairros das Indústrias e Expedicionários apresentaram entre 0,6 e 0,8 caso por mil habitantes. Todavia, salienta-se que $36,63 \%$ dos bairros da cidade de João Pessoa apresentaram baixa incidência (0 a 0,2 caso por mil habitantes do bairro) de casos registrados de violência doméstica contra a mulher entre os anos de 2003 a 2005, são eles: Mumbaba, Mussuré, Barra de Gramame, Distrito Industrial, Costa do Sol, Mussumagro, Aeroclube, Jardim Oceania e Miramar.

Para permitir a comparação entre bairros em um mesmo mapa, calcula-se o risco relativo para cada bairro em relação à cidade de João Pessoa. Apresentou-se sob a forma de mapas coropléticos a intensidade do risco de violência doméstica contra a mulher. O risco aumenta de forma proporcional à cor utilizada, ou seja, quanto mais forte a tonalidade maior o risco.

Segundo o mapa do risco relativo (Figura 2), os bairros que apresentaram os maiores riscos são: Bairro São José, Bessa, Mandacaru, Bancários, Centro, Mangabeira, Valentina, Ernesto Geisel, José Américo, Cristo Redentor, Funcionários, Alto do Mateus, Ilha do Bispo e Cruz das Armas. Apenas $21 \%$ dos bairros apresentaram baixo risco, dentre eles: Barra de Gramame, Mussuré, Mussumagro, Jardim Oceania, Aeroclube, Mumbaba, Varjão e Portal do Sol. Existem ainda, bairros que tiveram o risco entre 1,5 e 2,0 vezes

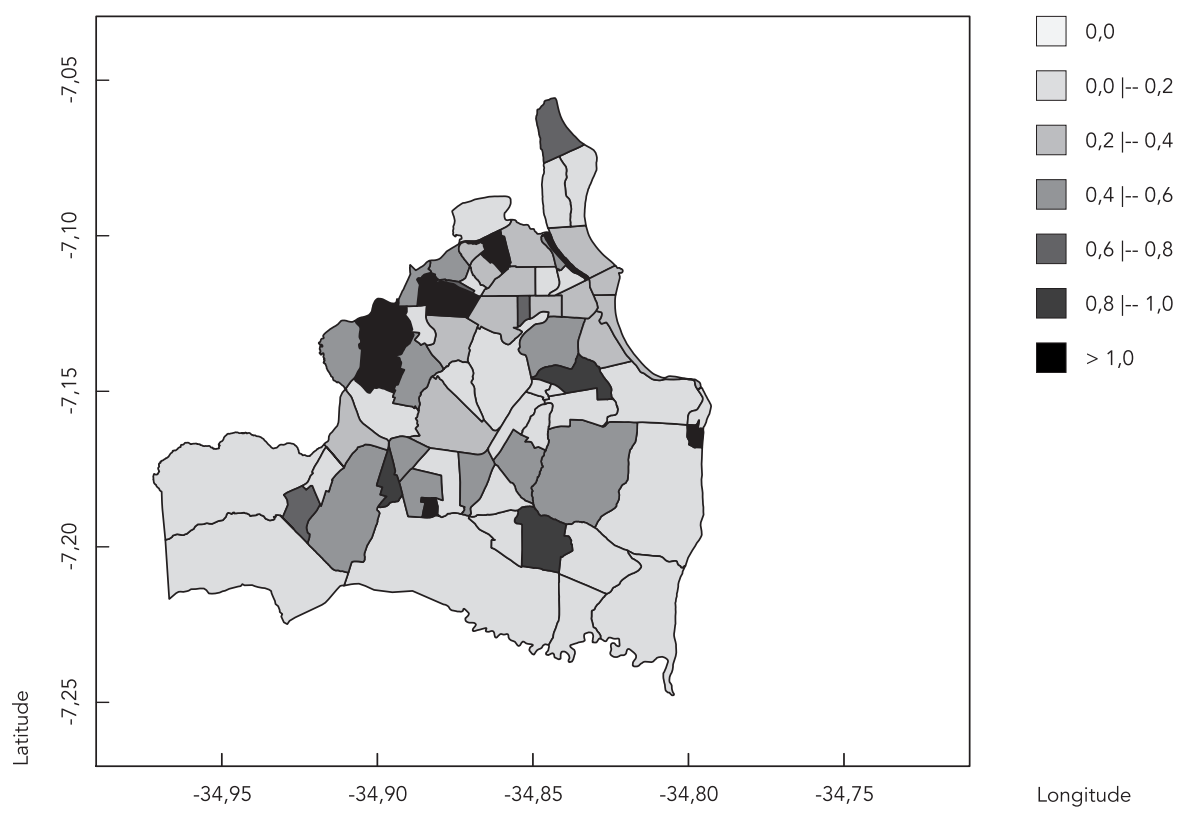




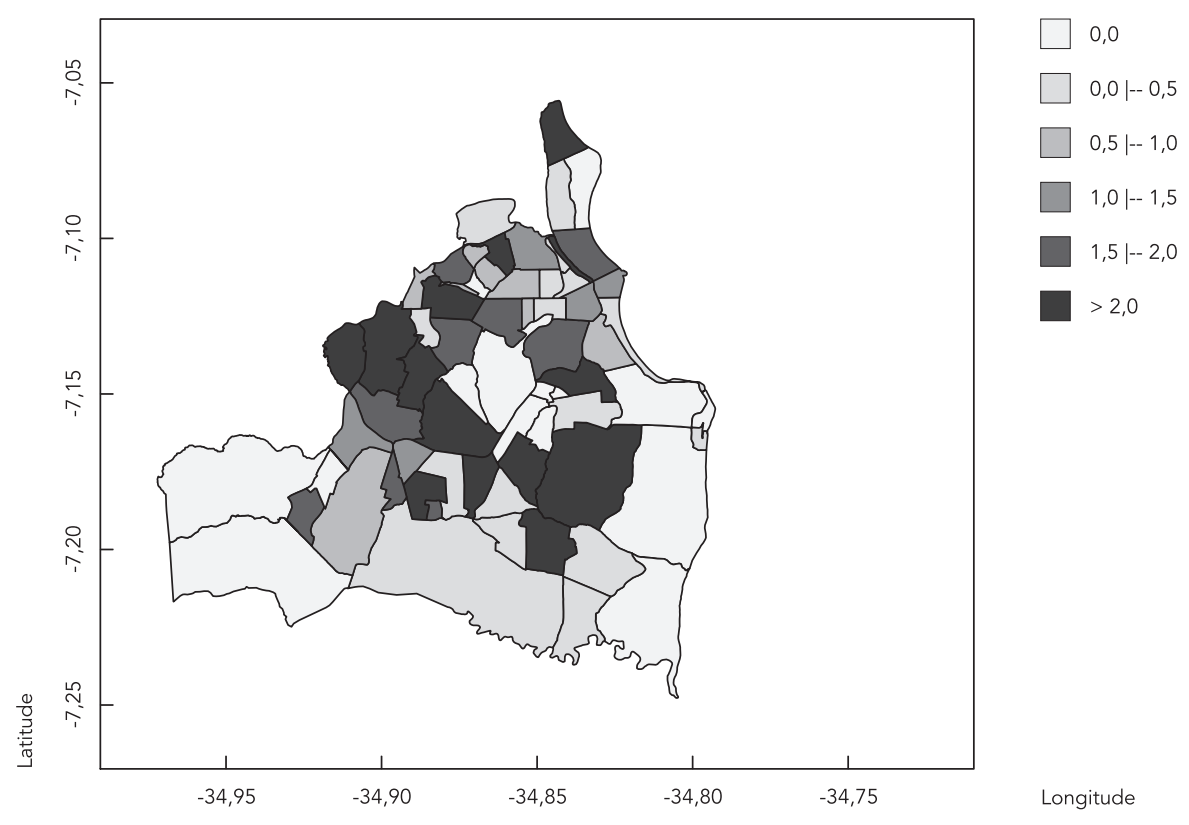

maior que a incidência da cidade de João Pessoa, como, por exemplo, Grotão, Oitizeiro e o Bairro das Indústrias.

Dentre as várias distâncias testadas para se verificar a significância dos resultados, o teste global de correlação espacial de Getis e Ord, para o ano de 2002, apresentou significância estatística para a distância 0,0195 (1,95km). Dessa forma, para o referido ano e distância é possível então calcular o teste local de correlação espacial de Getis e Ord. Observa-se no mapa de Getis e Ord local (Figura 3a), que todos os bairros apresentaram correlação negativa entre a violência doméstica contra a mulher e o espaço, existindo apenas um bairro com aglomerado positivo, Bairro do Cuiá, mas não significativo. Nos dados referentes ao ano de 2003 (Figura 3b), o índice de Getis e Ord para a violência doméstica contra a mulher obteve significância estatística para a distância $0,06(6,66 \mathrm{~km})$. Para esse ano estudado observa-se que a maioria dos bairros apresentou correlação negativa não significativa entre a ocorrência de violência doméstica contra a mulher e o espaço, porém três bairros apontaram aglomerados positivos, Expedicionários, Tambauzinho e Miramar, contudo, não significativo.
Para o ano de 2004 (Figura 3c), o índice de Getis e Ord apresentou significância estatística para a distância $0,06(6,66 \mathrm{~km})$. Observa-se que os bairros Tambiá, Expedicionários, Tambauzinho, Miramar, Jaguaribe e Trincheiras apresentaram correlação positiva entre a ocorrência de violência doméstica contra a mulher e o espaço, porém, não significativo. As demais regiões apresentaram correlação negativa não significativa.

A aplicação da estatística global de Getis e Ord apresentou aglomerados espaciais de violência doméstica contra a mulher para o ano de 2005 (Figura 3d) porque os valores eram maiores que o nível de significância de 0,05. Considerando as distâncias testadas de 0,01 (1km) e 0,15 (15km), foi observado que a distância a qual se obteve correlação espacial foi d = 0,0195 $(1,95 \mathrm{~km})$. Verifica-se a ocorrência de uma aglomeração espacial de valores positivos e significativos nos bairros de Tambiá e Tambuzinho, e de aglomerações de baixos valores significativos nas demais regiões da cidade. Os bairros assinalados foram os que apresentaram aglomerações com significância positiva. 
a) 2002

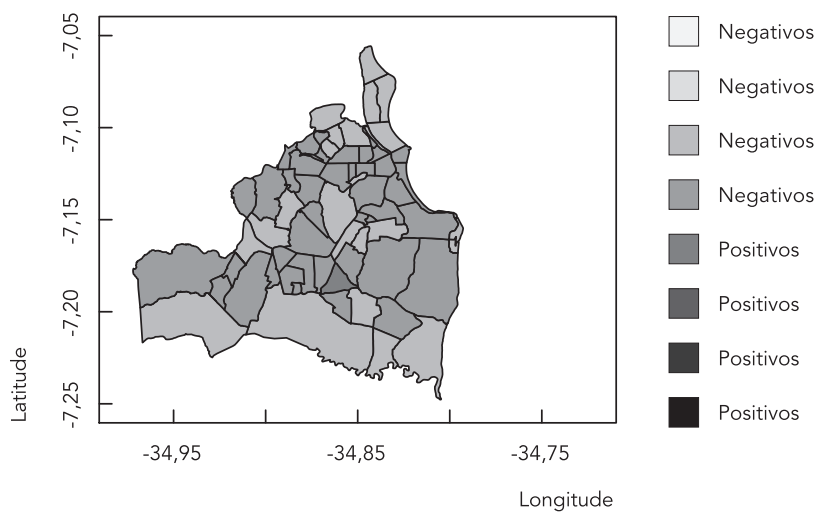

c) 2004

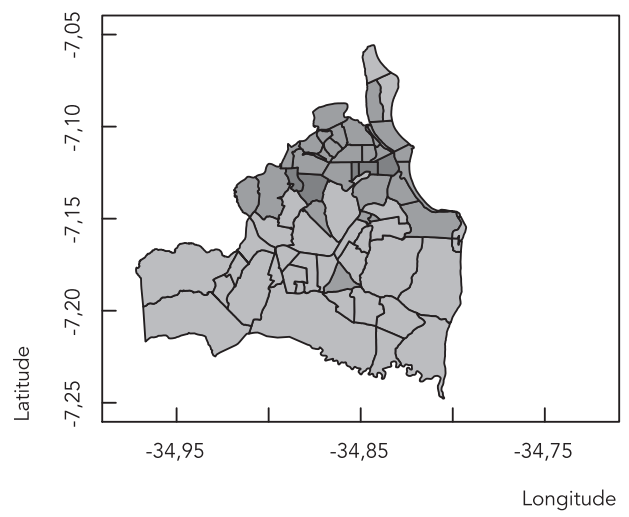

b) 2003

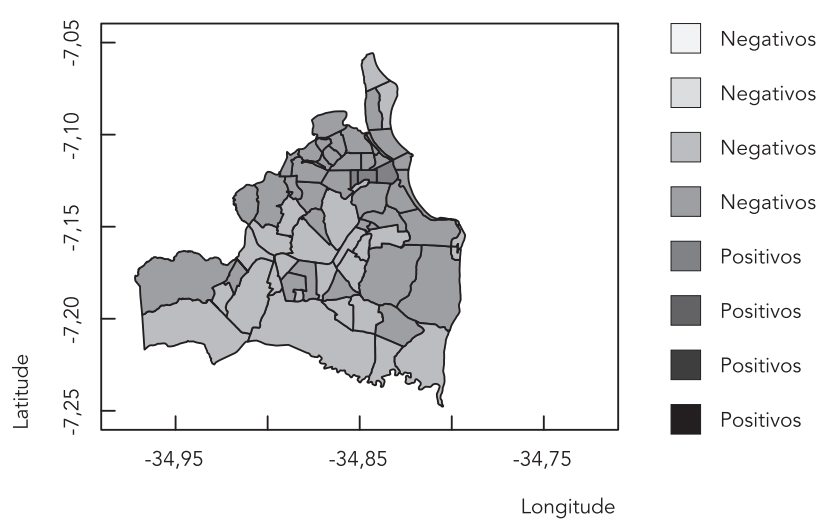

d) 2005

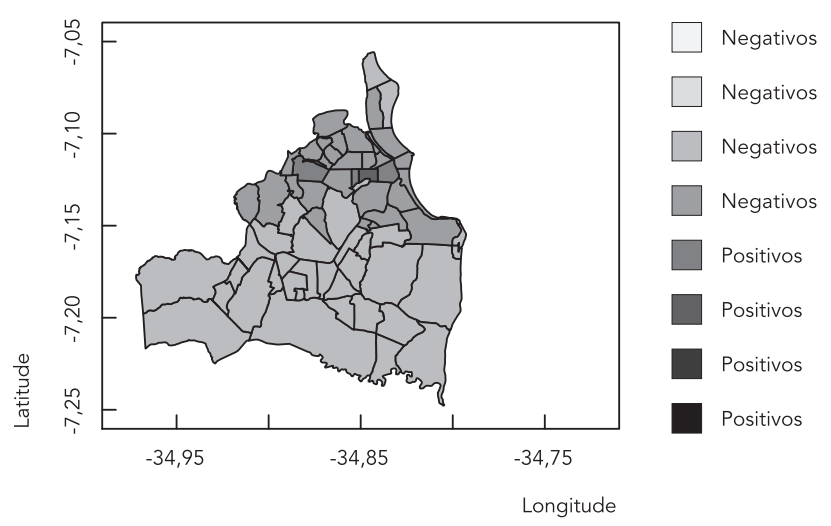

\section{Discussão}

As estatísticas da violência no Brasil, quando analisadas, podem auxiliar na identificação de padrões na distribuição espacial e temporal da violência, e pode levar os órgãos responsáveis pela segurança pública a tomar decisões no combate à violência. O mapeamento e a análise estatística são reconhecidos como poderosas ferramentas para o estudo e controle da violência urbana, uma vez que os mapas ajudam os gestores a identificar problemas na região de atuação. Considerando a grande carência de informações adequadas para a tomada de decisões sobre os problemas sociais, urbanos, rurais e ambientais, o geoprocessamento apresenta um enorme potencial para este auxílio, principalmente se baseado em tecnologias de custo relativamente baixo, em que o conhecimento seja adquirido localmente.

A especificidade de cada lugar pode ser entendida como uma valorização inerente a cada variável e como significação que se traduz em conceitos ${ }^{15}$. Isso significa que cada lugar atribui aos elementos constituintes do espaço uma relação valorativa (em relação ao universal) e um significado particular (em relação à totalidade social), conforme os estudos sobre as abordagens metodológicas de enfoque quantitativo e qualitativo 16,17 .

Nessa perspectiva, a cidade é um espaço geográfico e geopolítico onde se produzem e se 
aplicam os conceitos e outros instrumentos de trabalho com potencial conservador/transformador da realidade social. A elaboração e aplicação de políticas públicas são oportunidades e momentos para essa prática social. Tais situações comportam diferentes enfoques metodológicos e requerem o emprego de uma multiplicidade de ferramentas que contribuam para a definição de prioridades e ampliação do acesso da população aos bens e serviços públicos.

Entre tais ferramentas, destaca-se a relevância da utilização do conhecimento da área do geoprocessamento, especificamente o SIG, nos estudos sobre a distribuição espacial de problemas e análises de situações de saúde, como a violência doméstica contra a mulher, tema e parte do objeto deste trabalho. O SIG permite identificar as vulnerabilidades de grupos populacionais, informação de valor para o processo da tomada de decisão por ocasião da elaboração e adoção de políticas públicas, bem como para a planificação e gestão dos serviços e processos de trabalho, os quais organizam e executam o conjunto de intenções que compõem as políticas públicas.

Neste estudo, a utilização da análise espacial da violência doméstica contra a mulher revelou que não existe um padrão específico da violência de gênero. O fenômeno manifestou-se distribuído em todas as regiões territoriais do cenário investigado. Os resultados apontam a composição de mapas de incidência, do risco relativo e do índice de Getis e Ord, permitindo a produção de um modelo de decisão em saúde que pode ser aplicado por gestores no enfrentamento da violência doméstica, por identificar as áreas com maior risco de mulheres sofrerem danos físicos, emocionais e sociais, em decorrência deste tipo de violência.

O mapeamento das áreas de maior risco direciona a atenção dos poderes públicos, possibilitando uma ação eficaz por ser rápida e focalizada no nível local, com potencial ampliado, para centrar esforços nas piores condições identificadas. Isso favorece a racionalização dos recursos destinados para a alta, média e baixa complexidades, na atenção à saúde da mulher em situação de violência doméstica. De posse desse conhecimento, as medidas podem ser racionalmente escolhidas para o enfrentamento dos problemas na prática profissional, ampliando as chances de sua resolutividade.

Nesse enfoque, o enfrentamento dos problemas de saúde da mulher em situação de violência doméstica necessita da ação intersetorial e transdisciplinar, ou seja, os serviços precisam de comunicação eficaz entre si e de adequação de informações para selecionar seus conteúdos e objetivos. O envolvimento do território, nessa questão, permite potencializar as ações de saúde, sob a forma de práticas multiprofissionais, em contextos que requerem a intersetorialidade e o acompanhamento permanente, situações que se aplicam ao problema da violência de gênero.

Por essas razões, reafirma-se o argumento de que a prática profissional, para a elaboração e aplicação de políticas de enfrentamento da violência de gênero, que dispuser da microlocalização dos problemas e de informações confiáveis da geografia crítica, além dos instrumentos da estatística e da informática, poderá apoiar os profissionais no processo de tomada de decisão com maior possibilidade de eficácia e eficiência no atendimento das necessidades sociais, no tocante à saúde da mulher, em situação de violência de gênero.

O estudo também comprova um dos argumentos do referencial teórico de gênero: o de que a violência doméstica contra a mulher é um fenômeno que perpassa todas as classes sociais ou os diferentes segmentos sociais. Nesta investigação, a violência doméstica de gênero se apresentou em todos os bairros, indistintamente, porém houve aglomeração espacial significativa em dois bairros, conforme já apresentado nos resultados.

Assim, apesar da violência doméstica ser um fenômeno que atravessa todas as classes sociais, os riscos para a ocorrência do evento são maiores nos espaços geográficos de condições sociais menos favorecidas ou de maior desigualdade social. Neste trabalho, os bairros Ilha do Bispo, Centro, Mandacaru, Bairro São José, Penha e Grotão foram os de maior incidência e risco relativo. Esses bairros são habitados em sua maioria por pessoas com baixo poder aquisitivo. Segundo Barata 18, em todas as sociedades as situações de risco, os comportamentos relacionados à saúde e ao estado de saúde físico e mental tendem a variar entre os grupos sociais, em decorrência das desigualdades, categoria contemplada pela teoria da determinação social do processo saúde-doença.

A referida autora acrescenta que essa teoria é largamente empregada na América Latina, e sua versão, no Brasil, enfatiza o modo de vida. $\mathrm{O}$ enfoque engloba, além do espaço geográfico, aspectos materiais relacionados aos mecanismos de acumulação do capital, distribuição do poder, reprodução social e aspectos simbólicos, de modo que tais mecanismos indicam a posição de classe e a reprodução social como os principais determinantes do perfil de saúde e doença 18.

Os indicadores territoriais na construção do Mapa da Exclusão/Inclusão Social de São Paulo revelaram as expressões territoriais das desigualdades sociais. Propôs como dispositivo analítico 
do fenômeno estudos transdisciplinares, com aplicação de diversas linguagens metodológicas para análise das dinâmicas socioespaciais em múltiplas escalas, tendo como referência o território local, a cidade, porque a desigualdade social constitui um elemento básico para dimensionar indicadores territoriais no enfrentamento da questão social urbana, principalmente no estudo da relação exclusão/inclusão social e, consequentemente, para a elaboração de políticas públicas inclusivas 19,20.

Assim, a espacialização das informações sobre a violência doméstica, no cenário investigado, permite refletir sobre a necessidade de se qualificar os profissionais - com enfoque em conteúdos da abordagem de gênero - para atender a mulheres em situação de violência. Evidencia-se também, a necessidade de se equipar os serviços públicos de atenção, para atender as necessidades desse segmento populacional - como as Equipes de Saúde da Família, que são diretamente responsáveis pelo cuidado e compõem a rede do sistema de saúde da zona urbana de João Pessoa - uma vez que o fenômeno se encontra disseminado em bairros onde se concentram as classes sociais populacionais que mais utilizam os serviços públicos de saúde. O fato requer enfrentamento, desde a etapa do planejamento das políticas até sua realização, como trabalho de práticas multiprofissionais.

No sentido de superar o planejamento tradicional em saúde, o SIG se apresenta como um instrumento adequado para subsidiar um novo enfoque: o do planejamento estratégico em saúde, que pressupõe um sistema interligado de serviços que formam uma rede articulada, além disto, este sistema apresenta potencial para produzir informações que favorecem a resolutividade de problemas e necessidades de saúde no território 21.

O reconhecimento da violência contra a mulher como uma questão de saúde pública, por seu impacto nos âmbitos biológicos, sociais e psicológicos, que requer acesso a um tipo complexo de atenção e de serviços de saúde, exige o tratamento da questão sob enfoques que extrapolam os recursos tradicionalmente empregados pela área da saúde, requerendo a interseção da saúde com outras áreas do conhecimento humano.

Há uma corrente de pensamento sobre gênero que defende o argumento de que a condição de gênero é importante para os estudos dos perfis epidemiológicos, no que se refere às diferentes determinações e mediações que influenciam o processo saúde-doença, tendo em vista que "as relações de gênero atravessam todas as dimensões da vida social, possuem dinâmica própria, independente de outros processos sociais e são marca- das pelo antagonismo na relação de dominação das mulheres pelos homens" 18 (p. 77).

Embora a Epidemiologia Clássica tenha considerado a variável sexo como indicador de padrões de comportamentos específicos para homens e mulheres, esta disciplina não pondera sobre a complexidade das relações de gênero, assim, a variável sexo, dos estudos epidemiológicos tradicionais, não abrange as múltiplas facetas que o conceito de gênero encerra, sobretudo a que se refere à assimetria do poder entre os dois sexos. O poder é uma das categorias que mais contribui para a formulação do conceito de gênero e sua assimetria, e, nas relações entre homens e mulheres, é um dos fatores responsáveis pela desigualdade que autoriza a violência e impacta o estado da saúde física e mental de mulheres.

Nessa perspectiva, a utilização eficaz da ampla rede intersetorial na atenção à saúde da mulher - casas-abrigos, centros de referência e reabilitação, defensorias e delegacias da mulher, Secretaria de Políticas para as Mulheres, Secretarias de Saúde e de Planejamento - assume contornos relevantes para a garantia da integralidade e da efetividade do atendimento à mulher em situação de violência. Recorrer a esses instrumentos de enfrentamento depende de decisões quanto à necessidade de instalação, escolha adequada de lugar geográfico e geopolítico apropriado para sua constituição, que podem ser informados pelo SIG.

Nesse contexto, para a articulação dos serviços torna-se necessário a presença, em todas as instituições, de ações interligadas com outros serviços e atores sociais para instituir a escuta qualificada e a reflexão crítica, no que concerne à atenção integral das mulheres em situação de violência 22 , fortalecendo o processo de tomada de decisão.

\section{Conclusões}

Considerando o reconhecimento da responsabilidade do Estado, como instância definidora das políticas públicas, entre as quais as referentes à saúde da coletividade - que articula as práticas profissionais em saúde à superestrutura social a perspectiva do planejamento e da gestão, nessa área, assume lugar de destaque. Esse enfoque requer a consideração da interdisciplinaridade e pressupõe o emprego de mecanismos e instrumentos necessários para sua consecução, entre os quais se encontram o modelo de decisão e o SIG, respectivamente.

A recorrência a tecnologias e soluções inovadoras oferece suporte ao processo de tomada de decisão em saúde pública, na planificação e 
desenvolvimento de políticas públicas que buscam respostas efetivas, dirigidas à prevenção da violência doméstica contra a mulher e/ou controle de danos à saúde, para o melhoramento da qualidade de vida das pessoas e das condições sociais das cidades. Essa recorrência tem-se intensificado, sobretudo depois da ampliação do conceito de saúde e da situação jurídico-política da condição de ser a saúde um direito do cidadão e um dever do Estado brasileiro.

Tal interpretação da saúde permite destacar velhas práticas sociais como novos problemas de saúde, a exemplo da violência de gênero, um fenômeno secularmente praticado, mas somente na contemporaneidade constituído como um problema de saúde pública. Nesse contexto, os problemas de saúde requerem a apreciação dos múltiplos fatores no que se refere à sua gênese $\mathrm{e}$ desenvolvimento, envolvendo também as possi-

\section{Resumo}

Trata-se de um estudo quantitativo, ecológico, exploratório com utilização de técnicas de análise espacial e do Sistema de Informações Geográficas. A população foi composta por todas as denúncias de mulheres vítimas de violência doméstica, residentes no Município de João Pessoa, Paraíba, Brasil, no período de 2002 a 2005, na Delegacia Especializada de Atendimento da Mulher. Objetivou-se investigar a distribuição espacial da violência doméstica para subsidiar os gestores no processo de tomada de decisão. A análise dos resultados permitiu identificar as áreas de alta e baixa incidências de violência, como também o risco de cada bairro comparado à cidade de João Pessoa. A partir do índice de Getis e Ord produziu-se a decisão das áreas prioritárias para intervenção sobre a violência doméstica. Ressaltamos a necessidade de mudanças no que se refere à atenção às mulheres vítimas de violência e a articulação entre as instituições com promoção da educação permanente nos serviços, contemplando discussões sobre gênero e violência contra a mulher.

Violência Doméstica; Mulheres; Identidade de Gênero bilidades de se recorrer a múltiplos instrumentos de apoio para sua visibilidade e localização espacial, por meio de ferramentas computacionais no campo do geoprocessamento.

Além dos instrumentos precisos, as investigações em saúde de enfoque quantitativo não descartam a possibilidade de cotejar seus dados com o enfoque qualitativo, para considerar algumas categorias que enriquecem as explicações de seus resultados, por permitir revelar a gênese e as consequências dos fenômenos, sobretudo, quando o tema se refere a conteúdos complexos, como os que informam a violência doméstica ou de gênero.

Portanto, o estudo apresenta-se como uma contribuição ao debate para enfrentar um dos grandes desafios do planejamento e da gestão em saúde, o processo de tomada de decisão, no sentido de concretizar o SUS.

\section{Colaboradores}

K. D. T. Lucena foi responsável pela produção da introdução, referencial teórico, resultados, análise e considerações finais do artigo. A. T. M. C. Silva foi responsável pela aprovação final da versão a ser publicada. R. M. Moraes responsabilizou-se pela concepção e desenho do artigo. C. C. Silva contribuiu substancialmente na análise e interpretação dos dados. I. M. P. Bezerra responsabilizou-se pela redação do artigo. Todos os autores tiveram participação na revisão do manuscrito. 


\section{Referências}

1. Schraiber LB, d'Oliveira AF. Romper com a violência contra a mulher: como lidar desde a perspectiva do campo da saúde. Athenea Digital 2008; 14:229-36

2. Schraiber L, d'Oliveira AFPL, Portella AP, Menicucci E. Violência de gênero no campo da Saúde Coletiva: conquistas e desafios. Ciênc Saúde Coletiva 2009; 4:1019-27.

3. Organização Mundial da Saúde. Relatório mundial sobre violência e saúde. Genebra: Organização Mundial da Saúde; 2002.

4. Safiotti HIB. A mulher na sociedade de classes: mito e realidade. Petrópolis: Editora Vozes; 1976.

5. Departamento de Ações Programáticas Estratégicas, Secretaria de Atenção à Saúde, Ministério da Saúde. Política nacional de atenção à saúde da mulher: princípios e diretrizes. Brasília: Ministério da Saúde; 2004

6. Fonseca RMGS. Equidade de gênero e saúde das mulheres. Rev Esc Enferm USP 2005; 39 Suppl 4:450-9.

7 Guedes RN. Violência conjugal: problematizando a opressão das mulheres vitimizadas sob o olhar de gênero [Dissertação de Mestrado]. João Pessoa: Universidade Federal da Paraíba; 2006.

8. Garbin CAS, Garbin AJI, Dossi AP, Dossi MO. Violência doméstica: análise das lesões em mulheres. Cad Saúde Pública 2006; 22:2567-73.

9. Saffioti HIB. Gênero, patriarcado, violência. São Paulo: Fundação Perseu Abramo; 2004.

10. Correia VRM, Monteiro AMV, Carvalho MS, Werneck GL. Uma aplicação do sensoriamento remoto para a investigação de endemias urbanas. Cad Saúde Pública 2007; 23:1015-28.

11. Carvalho MS, Pina, MF, Santos SM. Conceitos básicos de sistemas de informação geográfica e cartografia aplicada à saúde. Brasília: Organização PanAmericana da Saúde/Ministério da Saúde; 2000.
12. Lucena KDT. Análise espacial da violência doméstica contra a mulher entre os anos de 2002 e 2007 em João Pessoa-PB [Dissertação de Mestrado]. João Pessoa: Universidade Federal da Paraíba; 2011.

13. Carvalho MS, Souza-Santos R. Análise de dados espaciais em saúde pública: métodos, problemas, perspectivas. Cad Saúde Pública 2005; 21:361-78.

14. Anselin L. Spatial data analysis with GIS: as introduction to application in the social sciences. Santa Barbara: National Center for Geographic Information and Analysis, University of California; 1992.

15. Santos M. Espaço e método. São Paulo: Edusp; 2008.

16. Minayo MCS. Violência: um problema para a saúde dos brasileiros. In: Ministério da Saúde, organizador. Impacto da violência na saúde dos brasileiros. Brasília: Ministério da Saúde; 2005. p. 9-42.

17. Turato ER. Métodos qualitativos e quantitativos na área da saúde: definições, diferenças e seus objetos de pesquisa. Rev Saúde Pública 2005; 39:507-14.

18. Barata RB. Como e porque as desigualdades sociais fazem mal à saúde. São Paulo: Editora Fiocruz; 2009.

19. Sposati A. Mapa de exclusão/inclusão social de São Paulo. São Paulo: Educ; 1996.

20. Sposati A. Cidade em pedaços. São Paulo: Editora Brasiliense; 2001.

21. Paim JS. Desafios para a saúde coletiva no Século XXI. Salvador: EDUFBA; 2006.

22. d'Oliveira AFPL, Schraiber LB, Hanada H, Durand J. Atenção integral à saúde de mulheres em situação de violência de gênero - uma alternativa para a atenção primária em saúde. Cienc Saúde Coletiva 2009 ; $14: 1037-50$.

Recebido em 03/Jul/2011

Versão final reapresentada em 13/Jan/2012 Aprovado em 02/Mar/2012 